\title{
SCHIZOPHRENIA, VITAMIN D AND RICKETS - IS THERE A RELATIONSHIP?
}

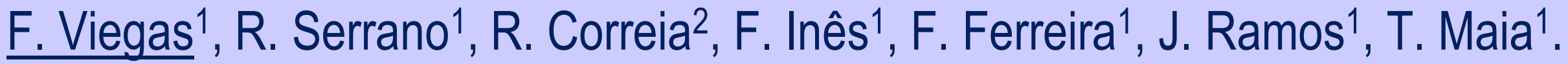 \\ ${ }^{1}$ Hospital Prof Doutor Fernando da Fonseca, Psychiatry, Amadora, Portugal. \\ ${ }^{2}$ Centro Hospitalar Universitário do Algarve, Psychiatry, Faro, Portugal.
}

\section{OBJECTJVES}

To review if there is any role of vitamin $D$ in the pathophysiology of schizophrenia, based on a clinical case.

\section{BA ACKGROUND AND AJJJS}

Vitamin $\mathrm{D}$ is a fat soluble vitamin as well as a hormone precursor that plays an important role in bone metabolism and seems to have some anti-inflammatory and immune-modulating properties ${ }^{1}$. Vitamin $D$ deficiency is a global issue, with $14 \%$ of the world population experiencing low vitamin $D$ levels ${ }^{1}$. This vitamin has been usually associated with bone disorders such as rickets, osteomalacia, and osteoporosis ${ }^{1}$

Vitamin $D$ is crucial not only for the osteoarticular system, but also for brain development and functioning. Given the hypothesis of the neurodevelopment of schizophrenia ${ }^{2}$, the importance of genetic and environmental factors (such as vitamin $D$ deficiency) in the etiopathogenesis of this disease is increasingly being highlighted.

Vitamin $D$ receptor is widely expressed in human brain being most abundant in the hypothalamus and substantia nigra ${ }^{3}$ and vitamin $D$ deficiency has been associated to a range of many neuropsychiatric diseases, such as depression, Parkinson disease, autism and schizophrenia ${ }^{2}$. One accepted theory is that low vitamin $\mathrm{D}$ during pre and perinatal periods imprints on several tissues, leading to an increased risk of certain diseases (in addition to genetic and environmental factors) $)^{2}$. Animal models have shown that vitamin $D$ receptors are directly involved in the regulation of dopaminergic associated genes expression, which in turn affect dopamine production ${ }^{1,2}$, but there might be other mechanisms involved.

The authors present a case of a patient diagnosed with rickets and late-onset schizophrenia, exploring the potential relationships between these diagnoses.

61-year-old female patient, with a diagnosis of Congenital Hypophosphatemic Rickets.

Two hospitalizations in Psychiatry, the first at 50 y.o. and the second at 61 y.o, manifesting

* Persecutory delusions

* Somatic passivity phenomena

* Cenesthetic hallucinations

* Functional and labor decline

- Social isolation

* No return to premorbid functioning

* Vitamin D level $10 \mathrm{ng} / \mathrm{ml}$ (>30 ng/ml)

The patient is being seen in psychiatric consultation with the diagnosis of schizophrenia and is stable under long-acting injectable antipsychotic and oral Colecalciferol (vit D3) suplementation

\section{WIATJERTALS AND JIJTHODS}

Clinical case description and non-systematic review of the literature published in the Pubmed / Medline database, using the following keywords: "schizophrenia", "psychosis", "rickets" and "vitamin D deficiency".

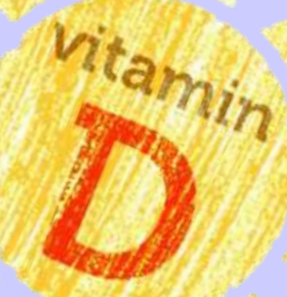

RESULTS AND CONCLUSIONS

The hypothesis that vitamin D deficiency is involved in the pathophysiology of schizophrenia correlates with the fact that individuals

born in winter and spring months,

living in countries at higher latitudes;

living in urban areas;

migrants at an early age to countries with colder climates are at greater risk of developing schizophrenia ${ }^{2,4}$.

The common denominator, according to the literature, is a lower maternal serum vitamin $\mathrm{D}$ concentration. The biological mechanism is most likely related to vitamin D's action on the regulation of inflammatory and immunological processes, consequently affecting the manifestation of clinical symptoms later in life ${ }^{1}$. If hypovitaminosis $D$ is extended to childhood, it can be predicted that children with rickets may have a higher risk of developing schizophrenia.

The etiologic basis of psychotic disorders remains largely unclarified, despite several proposed genetic, neurodevelopmental, and environmental causative factors ${ }^{4}$. According to the literature, more research will be needed to determine the role of vitamin $D$ deficiency as a mediator of psychosis. As with so many other presumed associations of vitamin $D$ and different maladies, there is still no clear consensus that vitamin $D$ improves or is related to mental health'. Given that most studies on this subject are cross-sectional, a causal relationship is difficult to determine.

Environmental risk modifying factors need special attention, as their further clarification may provide some preventive measures for the reduction of the severity of illness ${ }^{4,5}$. 